Extrait de G.A. Lehmann / R. Wiegels (Hrg.), Über die Alpen und über den Rhein... Beiträge zu den Anfängen und zum Verlauf der römischen Expansion nach Mitteleuropa, Berlin, De Gruyter, 2015.

Michel Reddé

\title{
Befunde und Erkenntnisse zu den römischen Militäranlagen am Oberrhein in augusteischer und tiberischer Zeit ${ }^{\star}$
}

Mit Bezug auf die berühmten, von Florus (II, 30) erwähnten castella Drusiana glaubte J.-J. Hatt, mehrere solcher Kastelle in der elsässischen Ebene lokalisieren zu können und zwar, von Süden nach Norden, in Basel, Kembs, Kunheim-Biesheim, Straßburg und Forstfeld ${ }^{1}$. Was Straßburg betrifft, so übernahm er lediglich eine alte Idee, die bereits von R. Forrer dargelegt wurde ${ }^{2}$ und die er sein ganzes Leben verteidigte, indem er sich vor allem auf die angebliche Datierung einer Inschrift eines Reiters der ala Petriana (CIL XIII 11605) stützte - willkürlich auf das Jahr 12-10 v. Chr. datiert - statt auf eine schlüssige, aus dem archäologischen Material gewonnene Untersuchung ${ }^{3}$. H. Schönberger hatte sich 1985 mit viel größerer Vorsicht geäußert: „Solange das Fundmaterial von Strasbourg nicht ausreichend bearbeitet ist, lässt sich wirklich Sicheres zu seinem Anfangsdatum nur mit Vorbehalt sagen. Im Augenblick sieht es aus, als ob es in der Zeit von Haltern beginnt, aber nicht früher“4 . Die jüngste Veröffentlichung des Straßburger Bandes der Carte Archéologique de la Gaule (CAG) hat leider nicht die Frage nach dem militärischen Ursprung der Stadt geklärt. Dieses hängt mit dem Prinzip dieser Reihe zusammen, die alte und neue archäologische Befunde in einer vorläufigen Bestandsaufnahme zusammenstellt, ohne auf die primäre Dokumentation einzugehen. Man muss daher die dort vertretenen Synthesen und verschiedenen, gelegentlich nebeneinander stehenden Interpretationen in diesem Band mit Vorsicht zur Kenntnis nehmen ${ }^{5}$.

Was andere Standorte in der elsässischen Ebene betrift, wie Forstfeld oder Kembs, so wissen wir heute nicht viel Neues. Im Fall von Kembs ist das Lager noch nicht lokalisiert, wenn auch ein Reitergrabstein und Militaria, darunter viele Teile von Pferdegeschirr, die Hypothese eines Militärpostens wahrscheinlich machen. Allerdings muss die Datierung für den Moment offen bleiben ${ }^{6}$. Für die Militärposten

\footnotetext{
^ Ich möchte Herrn Prof. Rainer Wiegels für die Übersetzung des Textes aus dem Französischen danken.

1 Hatt, L'Alsace celtique et romaine, S. 32.

2 Forrer, S. 6.

3 Zuletzt siehe: Hatt, Argentorate. Strasbourg, S. 11. Seit Conrad Cichorius, Ala, in : RE, I,1, 1894, 1244 und zuvor bereits Wilhelm Henzen, Rhein. Jahrb. XIII, 80 vermutet man, dass der Name dieser Einheit, weiterhin bekannt als Ala Gallorum Petriana mit diversen Beinahmen, vermutlich auf den Präfekten Germanicus, T. Pomponius Petra, zurückgeht, dessen Karriere von einer Inschrift aus Regium Lepidum gut bekannt ist (CIL XI, 969). Laut Demougin, Prosopographie ${ }^{\circ} 247$ ist es wahrscheinlich, dass dieser Teil seiner Karriere zwischen 11 und dem Tod des Prinzen datiert. Cf. Spaul, Ala², S.180-181. 4 Schönberger, Die römischen Truppenlager, S. 335.

5 Baudoux et al., CAG Strasbourg.

6 Zu der Stele: Wiegels, Ein Reitergrabstein; zu den Militaria: Fort, Militaria.
} 
von Ehl und Ungersheim-Thurwald, die H. Schönberger nennt, fehlt eine eingehende und moderne archäologische Untersuchung. Derselbe Autor vermutet auch - und zwar berechtigterweise -, dass eine augusteische Datierung für Limburg aus Mangel an Belegen nicht gesichert ist; er selbst bevorzugte eine spätere Datierung ${ }^{7}$. Weder er noch J.-J. Hatt konnten in ihrer Zeit die neuen Ergebnisse der Ausgrabungen von Oedenburg oder die grundlegende Studie von E. Deschler-Erb zum Domhügel von Basel kennen ${ }^{8}$. Die wichtigsten Ergebnisse dieser Untersuchung hatte ich selbst in einem Artikel von 2005 zusammengefasst ${ }^{9}$, doch mehrere neue Erkenntnisse erlauben uns heute, meine Schlussfolgerungen zu den Anfängen der römischen militärischen Okkupation in der elsässischen Ebene zu präzisieren.

Für Strasbourg selbst wurde das Material der frühen Schichten aus den alten Ausgrabungen von St. Martin neu untersucht, womit er teilweise einen bereits von $\mathrm{H}$. Schönberger geäußerten Wunsch erfüllte ${ }^{10}$. Bevor wir die wichtigsten Schlussfolgerungen dieser Studie zusammenfassen, müssen wir zunächst auf einige methodische Probleme eingehen. Abgesehen von den alten - zumeist zufälligen - Entdeckungen aus der Zeit von R. Forrer, wäre es illusorisch, die gesamten archäologischen Funde der Nachkriegszeit aus Straßburg im Detail zu analysieren. Obwohl sie in knapper Form „,veröffentlicht“ wurden, haben wir für die Ausgrabungen unter dem Dom und der rue du Sanglier im Jahr 1948 oder in der Kirche von Saint-Etienne in den Jahren 1948 und 1956 und in der Saint-Médard Gasse 1953 keine Dokumentation, die uns erlauben würde, den archäologischen Kontext, die Stratigraphie der Keramik und Münzen miteinander in Bezug zu setzen ${ }^{11}$. Der Grund liegt zweifellos in der von J.-J. Hatt seit 1948 verfolgten Methode, der eine Chronologie als gegeben voraussetzte anstatt die Datierung aus der Analyse des Materials herzuleiten, so dass a priori „Brandhorizonte“ mit wichtigen, in den Textquellen erwähnten historischen Ereignissen in Zusammenhang gebracht wurden ${ }^{12}$. Darüber hinaus wurde das Phänomen der „Weiterverwendung“ von J.-J. Hatt weitgehend ignoriert, wie J. Baudoux betont, der einen Großteil des keramischen Materials aus alten Grabungen neu untersucht hat, so dass die Schichten oft um 20-30 Jahre „verjüngt“ werden müssen im Vergleich zur traditionellen Datierung.

So enthällt die berühmte „augusteische“ Schicht aus der ruelle Saint-Médard Terra Sigillata aus der Zeit nach 30 n. Chr., und die sogenannte „Brandschicht von

7 Schönberger, S. 336.

8 Deschler-Erb, Der Basler Münsterhügel.

9 Reddé, Où sont passés les castella Drusiana, S. 69-87.

10 Martin, Les niveaux romains.

11 Ausgrabungen bei der Kathedrale und in der rue du Sanglier: Hatt, Le passé romain de Strasbourg. Ausgrabungen von Saint-Étienne : Hatt, Découverte et Hatt, Rapport provisoire sur les fouilles de 1956. Ausgrabungen in der ruelle Saint-Médard : Hatt, Les fouilles de la ruelle Saint-Médard. Die komplette Bibliographie findet sich in CAG 67/2.

12 Hatt, Le passé romain. 
70“ enthält Elemente aus domitianischer Zeit ${ }^{13}$. A.-M. Adam, der das angeblich prähistorische Material des Ortes untersucht hat, kam zu dem Schluss, dass dieses Material eher den ersten römischen Schichten zugeordnet werden muss und nicht einer hypothetischen keltischen Siedlung, für die es keine Belege gibt ${ }^{14}$.
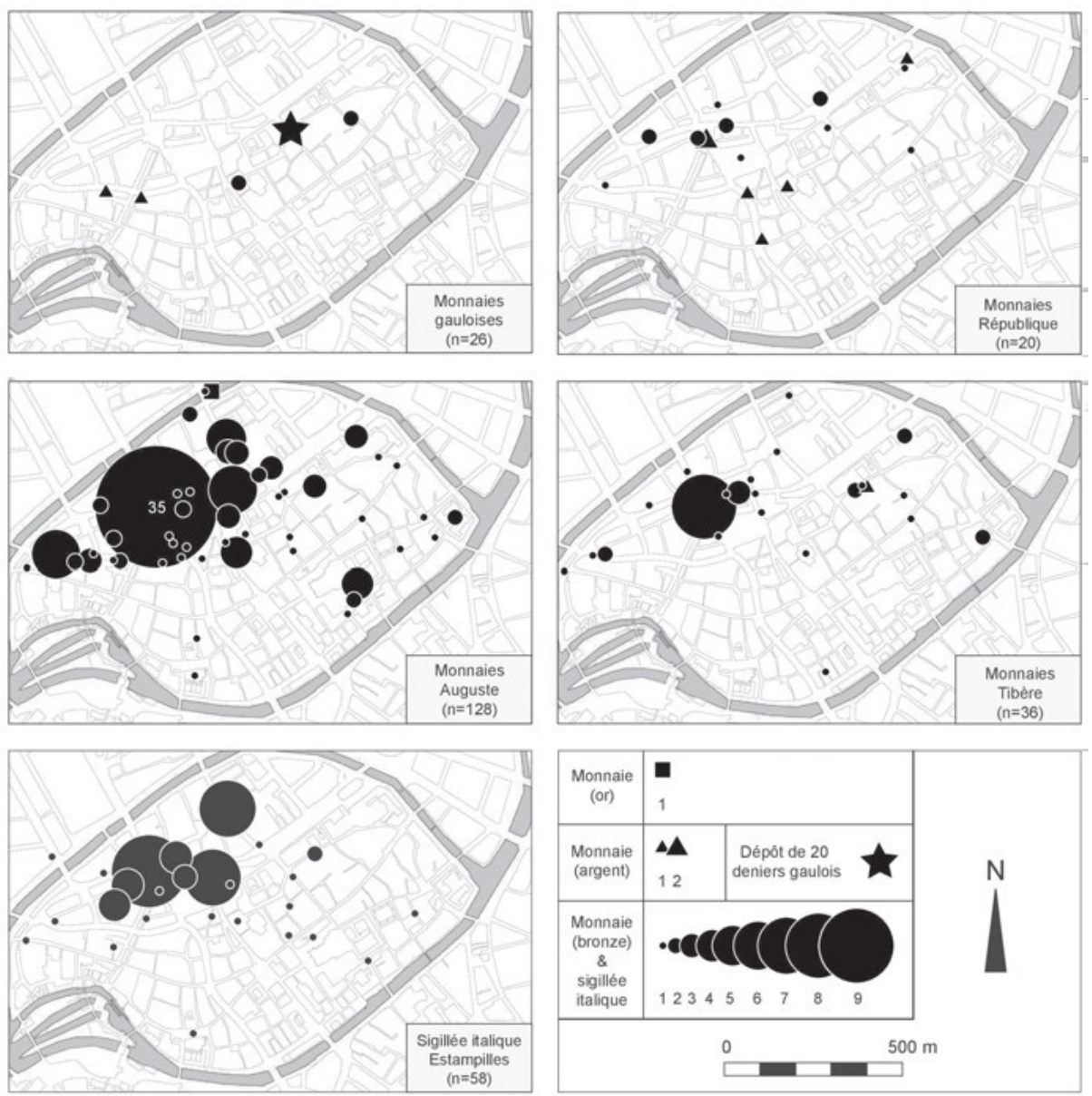

Abb. 1: Verortung des Materials der frühen Schichten in Strasbourg, nach St. Martin.

Unter diesen Bedingungen gibt es keine andere Lösung als die ältesten Fundzusammenhänge einen nach dem anderen mit Hilfe der wichtigsten Merkmale neu zu untersuchen, so wie es St. Martin gemacht hat, wohl wissend, dass diese Revision des Materials keine Wunder bringt. Diese Untersuchung, die so sorgfältig wie möglich am gesamten zur Verfügung stehenden Material durchzuführen war, erscheint mir

13 Baudoux, in : CAG 67/2, 74.

14 Anne-Marie Adam, Le secteur de Strasbourg à l'époque protohistorique, in : CAG 67/2, 54. 
dennoch sehr aufschlussreich. Sie erlaubt, einige grundlegende Fakten deutlich zu machen:

- Zum gegenwärtigen Zeitpunkt findet sich der früheste Befund nordwestlich vom zukünftigen Legionslager der VIII. Legion (Abb. 1).

- Der älteste numismatische Siedlungsbefund scheint nicht früher zu sein als allenfalls das Ende von Haltern und muss daher eher an den Beginn von Tiberius Regierungszeit datiert werden, obwohl eine solche präzise Angabe mit Vorsicht genossen werden sollte, da sie auf der Untersuchung eines relativ überschaubaren archäologischen Materials aus alten Ausgrabungen basiert (Abb. 2). Der Vergleicht der Münzhistogramme mit denen anderer früher tiberischer Orte, wie Velsen, Köln-Alteburg und Windisch-Legionslager, zeigt eine grosse Ähnlichkeit im Profil. Darüber hinaus sind etwa 38\% der Terra Sigillata Stempel Pisaner Herkunft, was charakteristisch für einen Horizont nach Haltern ist, während Töpfer von Arezzo Signaturen „in planta pedis“ fehlen; 60\% der Töpfer finden sich auch in Velsen I und Köln-Alteburg (Abb. 4).

- Nichts aber beweist bisher die Existenz von typischen militärischen Strukturen außerhalb des späteren Lagers der VIII. Legion. Es kann auch sein, dass sich das erste Lager von Argentorate nicht auf der Insel befand, wo später das Münster errichtet wurde, was der fest etablierten historiographischen Tradition widersprechen würde. Allerdings sollte man nicht die beiden Spuren einer Umwallung ignorieren, welche zum einen J.-J. Hatt in der rue Saint-Médard, zum anderen M.-D. Waton am Standort der ehemaligen Druckerei Istra beobachtet haben ${ }^{15}$ (Abb. 3). Im letzteren Fall wird eine tiberische Datierung vermutet, aber eine detaillierte Untersuchung des archäologischen Materials fehlt bisher. Anzumerken ist jedoch, dass keine dieser vermuteten Spuren von Umwallungen für die Unterbringung einer ganzen Legion ausreichen würde, während das Vorhandensein von Ziegeln und Inschriften der II. Legion deren Installation an diesem Ort vor Claudius' britannischen Feldzügen wahrscheinlich macht ${ }^{16}$.

Die jüngsten, von G. Kuhnle durchgeführten Ausgrabungen auf dem Gelände des ehemaligen Grenier d'Abondance und in der rue Brûlée bestätigen diese Annahmen ${ }^{17}$. Im ersten Fall haben sich die Recherchen auf einen Teil der Umwallung der achten Legion konzentriert. Sie belegen die Existenz einer ersten Holz-Erde-Mauer aus der Zeit nach Neros Regierungszeit und stammen wahrscheinlich aus den 90er Jahren. Aber die frühesten darunterliegenden Schichten scheinen nicht vor der Mitte des 1 . Jahrhunderts n. Chr. zu datieren - eine Chronologie, die durch die Ausgrabungen

15 Waton, Un nouveau système défensif à Strasbourg.

16 Reddé, L'histoire militaire de Strasbourg à la lumière des textes et des inscriptions, in : CAG 67/2, $110-113$.

17 Ausgrabungen INRAP 1999-2000 (Grenier d'Abondance) und 2008 (Rue Brûlée). Die beiden Ausgrabungsberichte sind nicht publiziert; eine Synthese ist in Bearbeitung durch G. Kuhnle. 
in der rue Brûlée, die sich auf eine zuletzt von den Baracken VIII. Legion besetzten Fläche konzentrierten, bestätigt wird.

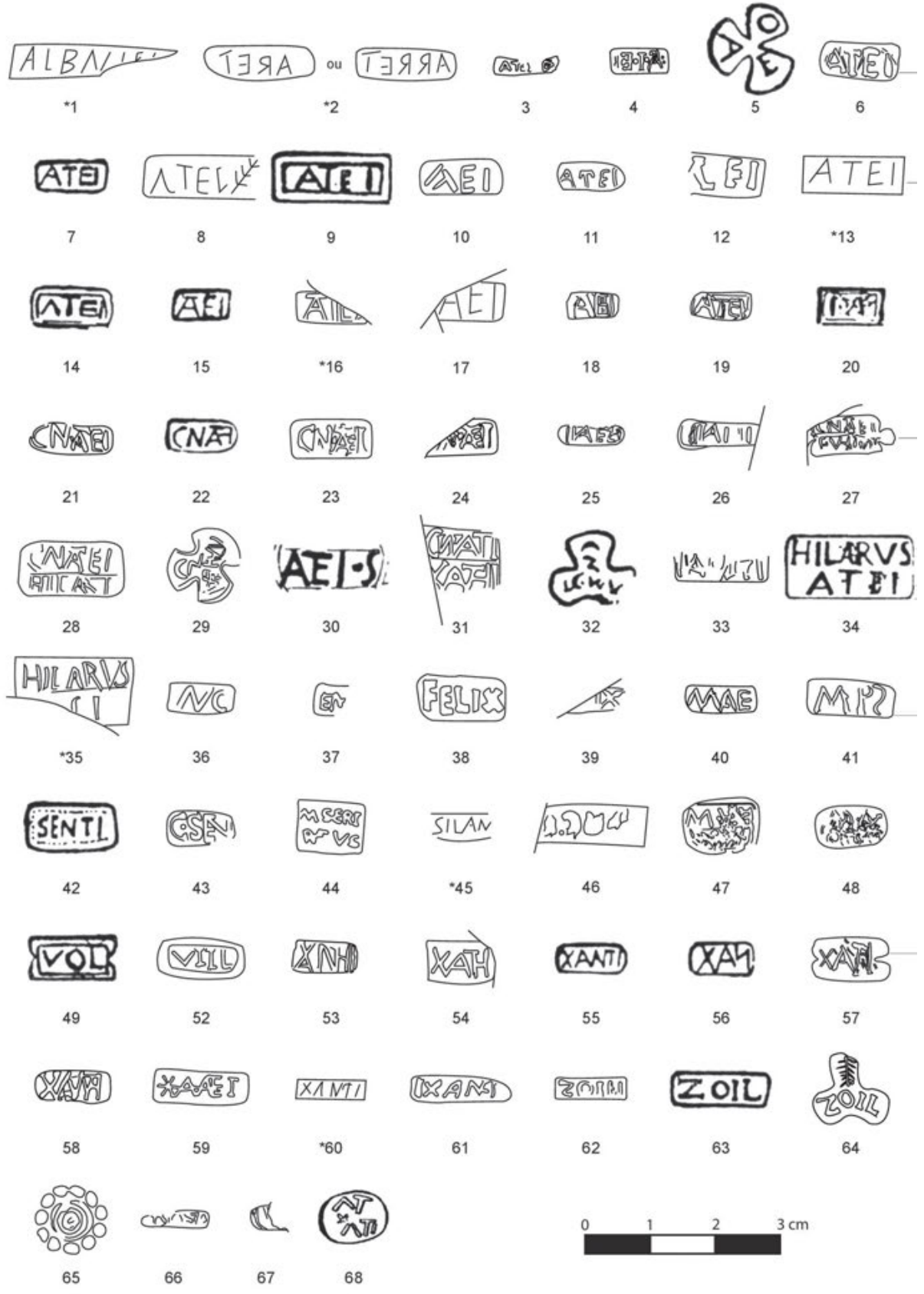

Abb. 2: Stempel auf Ziege $\overline{\overline{\bar{\nu}}}$ r frühen Schichten in Strasbourg, nach St. Martin. 

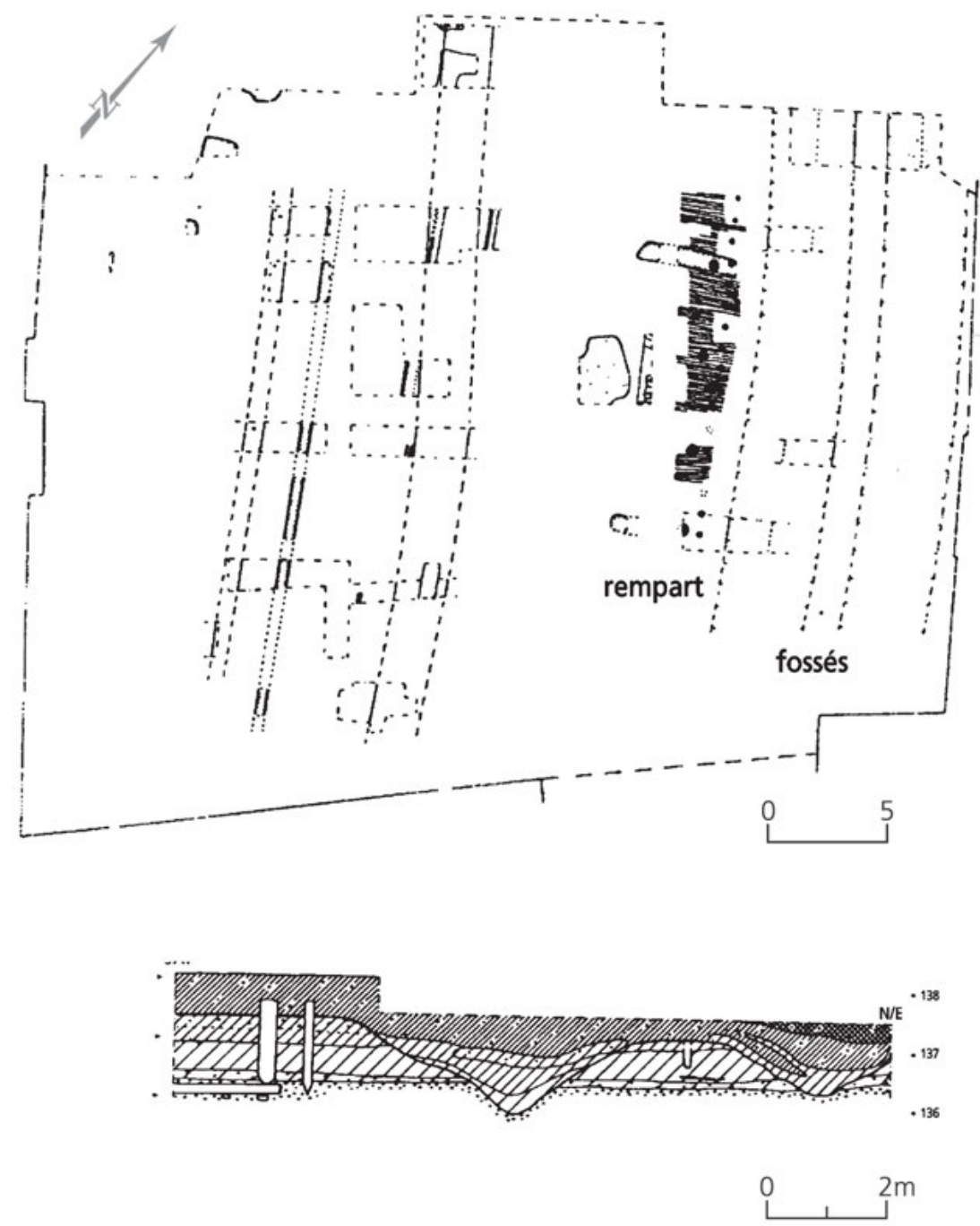

Abb. 3: Plan der Ausgrabung von Istra, nach M.-D. Waton.

Diese Ausgrabungsstätte befindet sich im Innern des Teiles der Umwallung, den M.-D. Waton am Standort der ehemaligen Druckerei Istra untersucht hat, was dazu einlädt, die topographischen und zeitlichen Beziehungen zwischen diesen beiden eng benachbarten Orten zu überdenken. Auch diese Untersuchung muss noch in Angriff 
genommen werden; sie würde eine detaillierte Analyse des aus den frühen Schichten der Ausgrabung von Istra stammenden Materials erfordern ${ }^{18}$.
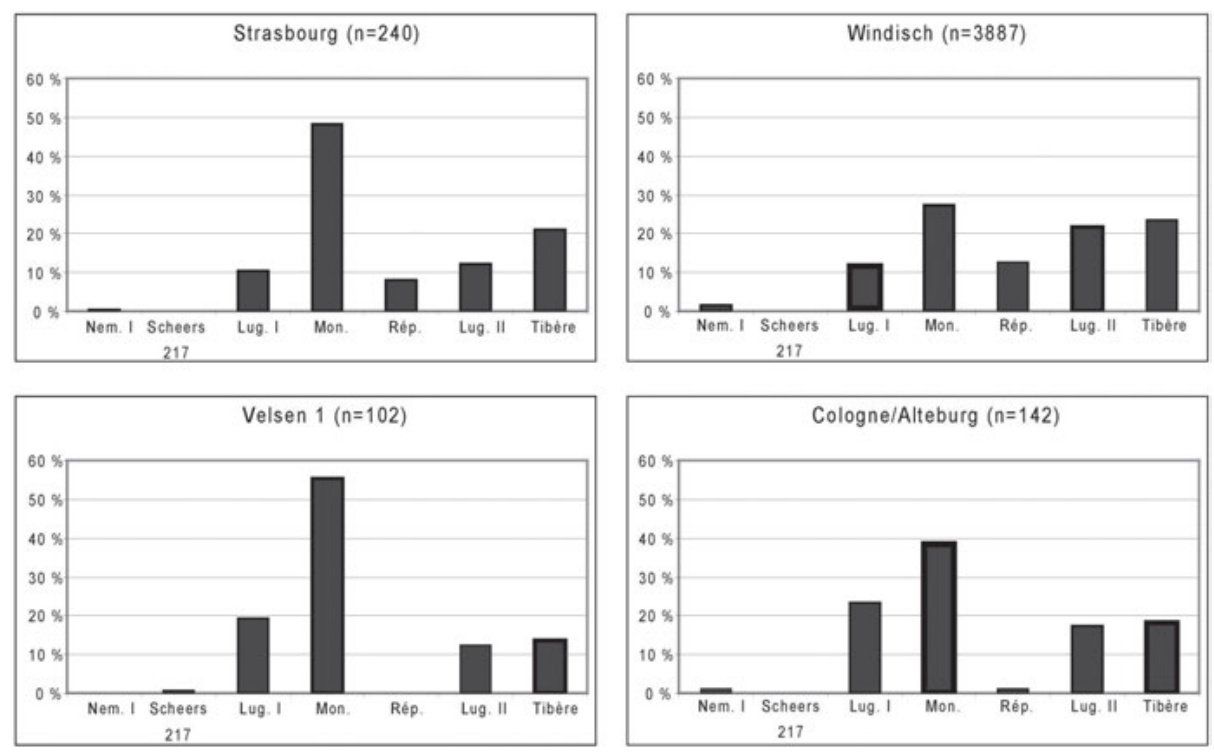

Abb 4: Vergleichende Diagramme der Münzen von Strasbourg, frühe Stufen, mit Windisch, Velsen 1 und Köln-Altenburg, nach St. Martin.

Die jüngsten Ausgrabungen von Oedenburg belegen ebenso eine militärische Besatzung, die nicht früher als in das zweite Jahrzehnt n. Chr. datieren kann. Die noch unveröffentlichten Forschungen aus dem Gebiet der zivilen Siedlung im Norden und Westen des Hügels Altkirch haben keine früheren Schichten aus vorrömischer oder augusteischer Zeit erwiesen, so dass die ersten sicheren chronologischen Indikatoren aus dem Gebiet der Heiligtümer und des Riedgrabens stammen (Abb. 5). Sie bestehen aus Holz, dessen Fälldatum um 3-4 n. Chr. liegt, und spätaugusteischer Keramik ${ }^{19}$. Diese allerersten Indikatoren, deren Chronologie durch die Numismatik bestätigt wird, scheinen sich gleichwohl auf den Bereich der Heiligtümer zu beschränken. Sie belegen eine Nutzung des Areals vor der Errichtung des ersten Militärlagers (B), das wir anhand von Keramik und Münzen auf die Jahre 15-20 n. Chr. datieren können ${ }^{20}$. Das Fällen einer großen Zahl an Bäumen im Frühjahr 19 n. Chr. markiert den systematischen Ausbau der Ufer des Riedgrabens, eine Arbeit, deren Ausmaß sicherlich eine

18 Ein willkommener Überblick zu den neuesten Forschungen findet sich im Ausstellungskatalog von Schnitzler / Kuhnle, Strasbourg-Argentorate.

19 Reddé (dir.) et alii, Oedenburg II, S. 101-117.

20 Reddé (dir.), Oedenburg I, S. 199-200, 243-248, 403-404. 
militärische Beteiligung anzeigt und somit ein Indiz für die Ansiedlung von Soldaten ist $^{21}$.

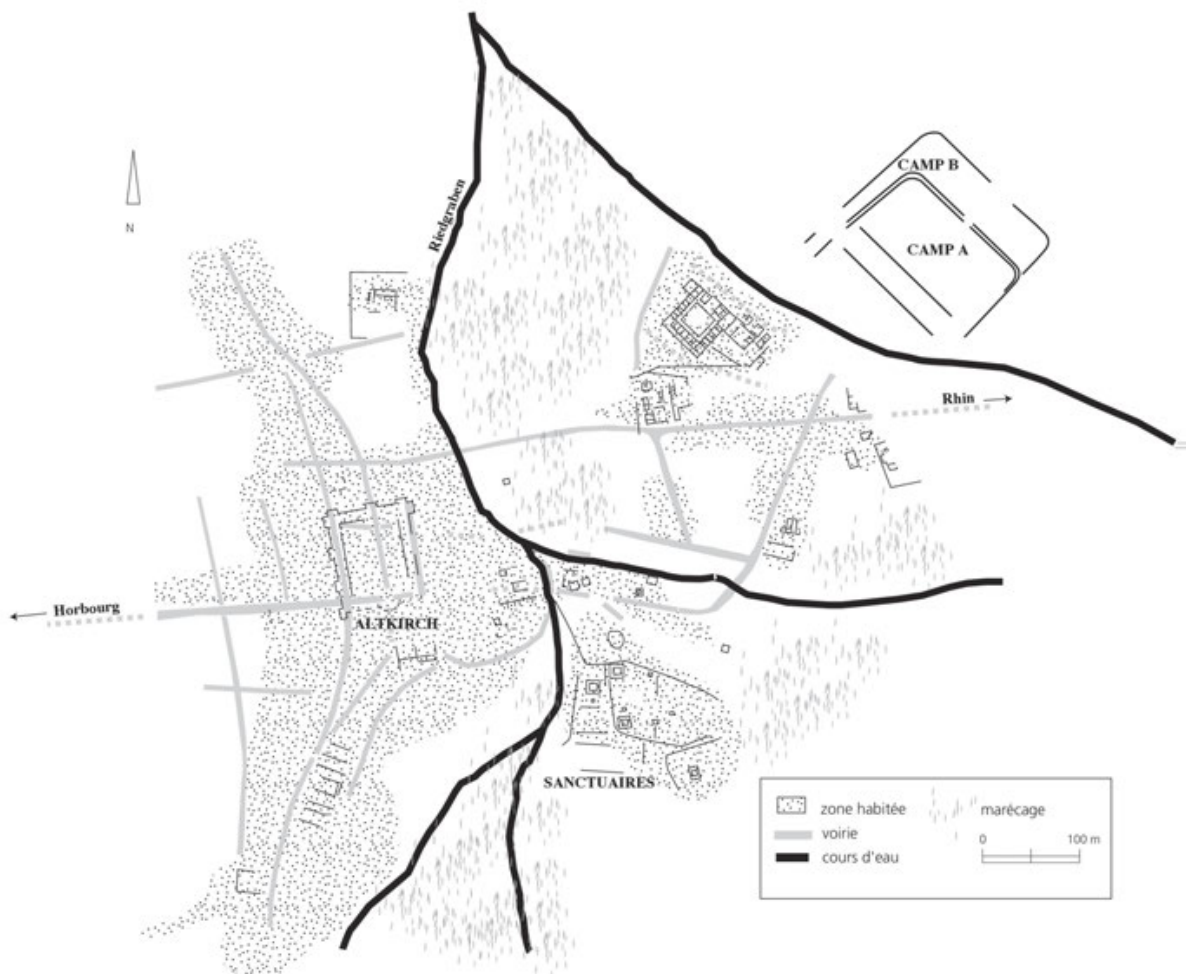

Abb. 5: Vereinfachter Übersichtsplan von Oedenburg, nach M. Reddé.

Das Lager B, das durch Lager A zerstört wurde, ist leider nur sehr unvollständig bekannt. Seine Dimensionen, zwischen 3,24 und 3,78 ha, sind untypisch sowohl für ein Legionslager als auch für ein Kohortenkastell. Man sollte daher in Betracht ziehen, dass es vielleicht eine Ala beherbergte, jedoch kann dies weder durch die Architektur der einen ausgegrabenen Baracke noch durch reichlich vorhandenes Pferdegeschirr oder einen epigraphischen Fund belegt werden. Die Metallobjekte sprechen genausogut für ein Lager einer gemischten Einheit aus Legionären und Hilfstruppen, was durchaus typisch ist für diese Epoche, auch wenn diese Vermutung nicht bewiesen werden kann. Bei der derzeitigen Befundlage muss deshalb ein endgültiges Urteil offen bleiben.

Das andere große Legionslager im oberrheinischen Becken ist Windisch, wo wir die chronologische Abfolge der ersten Anlagen durch verschiedene neuere Ausgra-

21 Reddé (dir.), Oedenburg II, S. 37-48. 
bungen besser verstehen können, und zwar vor allem durch die Ausgrabungen von Windisch-Breite und des keltischen Walles. Letzterer, offenbar um oder kurz nach $50 \mathrm{v}$. Chr. erbaut ${ }^{22}$, wurde wohl beim Bau des Legionslagers eingeebnet, was A. Hagendorn um $14 \mathrm{n}$. Chr. datiert ${ }^{23}$. Wir haben es ab dieser Zeit wohl mit der ganzen XIII. Legion zu tun. Vor dieser Periode war die Situation eine andere: Die Analyse des archäologischen Materials zeigt trotz des Fehlens von epigraphischen und literarischen Quellen deutlich, dass kleine militärische Abteilungen seit dem zweiten Jahrzehnt v. Chr. in Vindonissa anwesend waren, und zwar sowohl in dem Oppidum selbst als auch am Fuße desselben ${ }^{24}$.

Diese allererste augusteische Phase, vor der Gründung des so bezeichneten „Schrägen Lagers“ findet ihre Parallele in Basel auf dem Domhügel. Nach einer detaillierten Studie des gesamten an diesem Ort ausgegrabenen Materials hat E. DeschlerErb im Horizont III, der um 30-25 v. Chr. beginnt, die Präsenz regulärer Soldaten der römischen Armee nachgewiesen, und zwar vor allem für die Zeit von Dangstetten und der Alpeneroberung. Diese Soldaten, deren Anzahl relativ begrenzt war, haben sich mit der Zivilbevölkerung vermischt, da sie, obwohl der späteisenzeitliche murus gallicus geschliffen worden war, kein Lager im eigentlich Sinn hatten (dafür gibt es jedenfalls keine Belege) ${ }^{25}$. Man kann sich natürlich fragen, ob wir das gleiche Muster der Besatzung nicht auch in Limburg wiederfinden, einem kleinen, rechtsrheinischen Oppidum nördlich des Kaiserstuhls, und ob diese Form der „militärischen Präsenz“ inmitten der indigenen Bevölkerung nicht in dieser Region eine wichtige Phase vor der Installation von großen Militäreinheiten darstellte ${ }^{26}$. Allgemeiner gesagt, verweist das auf eine bereits von J. Metzler formulierte Hypothese zur Militärpräsenz in der Zeit nach Caesars Eroberung von Gallien und auf die Situation, die wir heute auf dem Titelberg erkennen können ${ }^{27}$. Das könnte auch als konzeptioneller Rahmen dienen, um die vielen römischen Militaria in vorrömischen Oppida zu erklären, wie M. Poux gezeigt hat ${ }^{28}$.

Wir müssen noch andere Faktoren kurz in Erinnerung rufen, bevor wir eine Synthese wagen: die mögliche Installation eines castellum vor den Toren der römischen Kolonie Augst unter Tiberius ${ }^{29}$ und den Militärposten Konstanz, der um 20 gegründet wurde ${ }^{30}$. Es ist nicht notwendig, auf diese mittlerweile gut bekannten Nachweise weiter einzugehen. Nützlich erscheint es jedoch, an das fast völlige Fehlen von archäo-

22 Pauli-Gabi, Ausgrabungen.

23 Hagendorn et alii, Zur Frühzeit von Vindonissa, S. 464-465.

24 Flück, Östlich des « Keltengrabens”.

25 Deschler-Erb, Der Basler Münsterhügel, S. 239-247.

26 Wendling, Sasbach am Kaiserstuhl.

27 Metzler, Das treverische Oppidum, S. 519-528.

28 Poux, Sur les traces de César. Ich habe meine eigene Interpretation im Nachwort dieses Werkes gegeben.

29 Deschler-Erb et alii, Das frühkaiserzeitliche Militärlager.

30 Mayer-Reppert, Römische Funde. 
logischen Fundorten in der elsässischen Ebene zu erinnern, die für die augusteische Zeit charakteristisch sind. Auf Grund der Analyse des gesamten keramischen Materials der Spätlatènezeit und der frühen römischen Besatzungszeit konnte M. Zehner das Überdauern von prähistorischen Elementen bis in die frühe Regierungszeit des Tiberius feststellen ${ }^{31}$. Es gibt jedenfalls nur wenige Siedlungen, die früh, also noch vor dem Beginn des 1. Jh. n. Chr., „romanisert“ wurden ${ }^{32}$, und die aktuellen, noch unveröffentlichten umfangreichen Ausgrabungen des Vicus Horbourg-Wihr widersprechen dieser Beobachtung nicht ${ }^{33}$, ebensowenig jene von Oedenburg. Man muss eine detailliertere Analyse der jüngsten Forschungen der verschiedenen archäologischen Teams von Brumath abwarten, um eine bessere Vorstellung von der Entwicklung der Hauptstadt der Triboker zu gewinnen. ${ }^{34}$ In Bezug auf ländliche Siedlungen erkennt man mancherorts (z.B. Houssen, Vendenheim, Ensisheim, Benfeld und Sierentz) eine Siedlungskontinuität zwischen der Spätlatène- und der Römerzeit, aber ohne eine deutliche Veränderung der materiellen Kultur oder der Siedlungsformen ${ }^{35}$. Obwohl archäologische Forschungen seit langem in dieser Region vernachlässigt wurden, können unsere offensichtlichen Wissenslücken alleine nicht den Gesamteindruck einer „verzögerten Romanisierung“ erklären.

Können wir anhand dieser archäologischen Evidenz eine historische Skizze von Roms frühen militärischen Dispositionen im oberrheinischen Becken erstellen? Es scheint mir heute ziemlich offensichtlich, dass wir keine Belege haben, die eine dauerhafte Stationierung regulärer Einheiten unterhalb von Basel vor dem Ende von Augustus' Regierungszeit beweisen können. In Basel selbst und in Windisch scheint die Anwesenheit von Soldaten, vermutlich ab dem Horizont von Dangstetten, gut belegt zu sein, aber das war eine Installation kleiner Abteilungen an der Seite der Zivilbevölkerung in einem von einheimischer Kultur und Gesellschaftsstrukturen geprägten Umfeld. Ob es sich um Legionäre oder Hilfstruppen oder um beide handelt, wird noch reichlich Stoff für Diskussionen bieten. Wir stellen fest, dass die Gründung der ersten echten Römerlager erst mit dem Regierungswechsel von Augustus zu Tiberius kurz vor oder kurz nach $14 \mathrm{n}$. Chr. erfolgte. In Straßburg übrigens müssen wir zweifellos das angeblich ,augusteische“ Lager der ala Petriana im Zentrum der Stadt

31 Zehner, La céramique de la Tène finale.

32 Flotté et alii, Les agglomérations antiques d'Alsace.

33 Flotté, Horbourg-Wihr.

34 Die Ausgrabungen, die 2009 in der Rue Rampont unter der Leitung von P. Flotté (PAIR) stattfanden, haben ergeben, dass die ersten datierbaren Schichten vom Ende des letzten vorchristlichen bis zum Beginn des 1. Jh. n. Chr. reichen. Eine Ausstellung, die vor kurzem im Museum in Strasbourg stattgefunden hat und die sich mit Brumath beschäftigte, zeigte die neuesten archäologischen Befunde, aber die Datierung der ersten Schichten wurde nur mit ,augusteisch' ohne weitere Spezifizierung angegeben (Schnitzler 2015, 90). Der Bestand an Lagern (Marschlagern?), der auf Luftbildaufnahmen in der Umgebung des Ortes beobachtet wurde, ist nicht datiert (ibid., 58-59).

35 Zehner, Habitats de plaine. 
vergessen; bislang fehlt jedenfalls jeglicher sachlicher Nachweis für eine solch frühe Befestigung.

Das bringt uns zurück zur Analyse der wenigen verfügbaren historischen Quellen, die uns jedoch keine Präzisierung der Chronologie erlauben, wie dieses bisweilen behauptet wurde. Dies ist meist eine Frage der Interpretation: Die berühmte Passage, in der Tacitus (Ann. I, 37) die Revolte nach Augustus“ Tod beschreibt, zeigt, dass die II. und die XIII. Legion einen Teil der „oberen Armee“ waren, aber das bedeutet nicht, dass sie bereits in ihrem späteren, permanenten Lagern stationiert waren, die eine in Straßburg ${ }^{36}$, die andere in Windisch ${ }^{37}$. Dennoch ist diese Interpretation nicht völlig von der Hand zu weisen, wenn man den materiellen Befund und die frühesten Gebäudespuren in Windisch bedenkt, wie von A. Hagendorn vorgeschlagen wurde. Letztere nimmt an, dass die Truppen mit dem Bau des Lagers vor Augustus‘ Tod begonnen hätten, diesen dann unterbrochen und erst viel später, vermutlich $16 \mathrm{n}$. Chr., nach den Offensiven des Germanicus, fortgesetzt hätten ${ }^{38}$. Diese Hypothese ist nicht abzuweisen, aber sie hängt natürlich von Faktoren ab, die ebenso unsicher wie subtil sind. Man vergisst in diesem Zusammenhang leicht, dass die militärische Situation in diesem Teil des Reiches ganz anders war als in der Germania Inferior. Die dauerhafte Installation von großen permanenten Einheiten an den Ufern des Rheins südlich von Straßburg resultiert wahrscheinlich nicht aus einer gravierenden Bedrohung. Wir sehen außerdem zehn oder fünfzehn Jahre nach der Gründung Oedenburgs die ersten Villen in der badischen Ebene ${ }^{39}$, also östlich des Rheins, und es ist unbestreitbar, daß die militärische Präsenz in einer Region, die bisher kaum romanisiert war, die Transformation der lokalen Gesellschaften beschleunigte. Es ist in diesem Zusammenhang nicht erforderlich, diese Ereignisse im Sinne einer „Großen Strategie“ und als Ergebnis der politischen Umwälzungen nach Tiberius‘ Regierungsantritt zu deuten. In diesem Teil Obergermaniens, wo die indigene Bevölkerung östlich des Rheins ziemlich spärlich war ${ }^{40}$, war die potenzielle Bedrohung für die römische Welt überhaupt nicht vergleichbar mit der Situation in Niedergermanien. Um die römische Militärpolitik und die entsprechenden Dispositionen südlich von Mainz besser einschätzen zu können, wäre es wichtig, die frühe Entwicklung von Worms ${ }^{41}$ und Speyer ${ }^{42}$ genauer zu verstehen. Auch hier ist die kritische Analyse der archäologischen Hinterlassenschaft aus der Frühzeit der militärischen Okkupation dieser Region ein wichtiges Desiderat der archäologischen Forschung.

36 Diese Belegung wurde aus sechs Grabinschriften (CIL XIII 5975-5978, 11628, AE 1998, 983) und einen Graffito auf einem Ziegel erschlossen (R. Forrer, Strasbourg-Argentorate, S. 282).

37 CIL XIII, 5206 ; Speidel, Die römischen Schreibtafeln, $n^{\circ} 5$.

38 Hagendorn, Zur Frühzeit, S. 464-465.

39 Insbes. jene von Heitersheim; cf. Seitz, Villen.

40 Wieland, Die Spätlatènezeit, S.238.

$41 \mathrm{Zu}$ Worms, s. jetzt Grünewald, Unter dem Pflaster, S. 32-41.

$42 \mathrm{Zu}$ Speyer, s. Bernhard, Militärstationen. 


\section{Literatur}

Baudoux, Juliette / Flotté, Pascal / Fuchs, Matthieu / Waton, Marie-Dominique : Strasbourg 67/2, Carte archéologique de la Gaule, Paris, 2002 (= CAG).

Bernhard, Helmut : Militärstationen und frührömische Besiedlung in augusteich-tiberischer Zeit am nördlichen Oberrhein, in : Studien zu den Militärgrenzen Roms III. 13. Internationaler Limeskongress, Aalen 1983. Forsch. u. Ber. BW, 20, 1986, S. 105-121.

Demougin, Ségolène : Prosopographie des chevaliers romains julio-claudiens, 1992, Coll. EFR, Rome, 153.

Deschler-Erb, Eckard / Peter, Markus / Deschler-Erb, Sabine : Das frühkaiserzeitliche Militärlager in der Kaiseraugster Unterstadt. Forschungen in Augst, 12, Augst, 1991.

Deschler-Erb, Eckard : Der Basler Münsterhügel am Übergang von spätkeltischer zu römischer Zeit. Ein Beispiel für die Romanisierung im Nordosten Galliens (mit Beitägen von Stopp, Barbara / Rentzel, Philippe / Thirion-Merle, Valérie/ Thierrin-Michael, Gisela). Materialhefte zurArchäologie in Basel, 2011, 22A u. B.

Fischer, Franz : Südwestdeutschland im letzten Jahrhundert vor Christi Geburt, in : Planck, Dieter (Hg.), Archäologie in Württemberg (Stuttgart 1988) 238.

Flotté, Pascal (dir.) : Horbourg-Wihr, Haut-Rhin. Kreuzfeld (est). Un quartier de l'agglomération gallo-romaine, Rapport final d'opération, 2012.

Flotté, Pascal / Fuchs, Matthieu / Wolf, Jean-Jacques / Herrgott, Matthias : Les agglomérations antiques d'Alsace, in : Bilan scientifique, hors série, 2/2, DRAC Alsace, 2006, S. 31-41.

Flück, Matthias : Östlich des « Keltengrabens ». Auswertung der Grabung Windisch-Dorfschulhaus 1986/87, in : Jahresber. Ges. Pro Vindonissa 2007, S. 17-57.

Forrer, Robert : Strasbourg-Argentorate : préhistorique, gallo-romaine et mérovingienne, Strasbourg, 1927.

Fort, Bérangère : Militaria et occupation militaire de l'agglomération secondaire de Kembs-Cambete, in : Rev. Arch. Est et Centre-Est, 52, 2003, S. 373-402.

Grünewald, Mathilde : Unter dem Pflaster von Worms. Archäologie in der Stadt, Worms, 2012.

Hagendorn, Andrea / Doppler, Hugo / Huber, Adrian / Hüster Plogmann, Heide / Jacomet, Stefanie / Meyer-Freuler, Christine/ Pfäffli, Barbara / Schibler, Jörg : Zur Frühzeit von Vindonissa. Auswertung der Holzbauten der Grabung Windisch-Breite 1996-1998. Veröffentlichungen der Gesellschaft Pro Vindonissa XVIII, 2003.

Hatt, Jean-Jacques : Le passé romain de Strasbourg. Stratigraphie chronologique, in : Gallia 7, 1949-2, 161-194.

Hatt, Jean-Jacques : Découverte de vestiges d'une caserne romaine dans l'angle du castrum d'Argentorate. Rapport provisoire sur les fouilles de l'Église Saint-Étienne à Strasbourg (été 1948), in : Cahiers Arch. et Hist. Alsace 1949, S. 257-276.

Hatt, Jean-Jacques : Les fouilles de la ruelle Saint-Médard à Strasbourg, in : Gallia 11, 1953-2, S. $225-248$.

Hatt, Jean-Jacques : Rapport provisoire sur les fouilles de 1956 sous l'Église Saint-Étienne à Strasbourg. Découverte d'une abside romaine du Ve siècle, in : Cahiers Arch. et Hist. Alsace 1958, S. 27-46.

Hatt, Jean-Jacques : L'Alsace celtique et romaine. 2200 av. JC à 450 ap. JC, Wettolsheim, 1978.

Hatt, Jean-Jacques : Argentorate. Strasbourg, Presses Universitaires de Lyon, 1993.

Martin, Stéphane : Les niveaux romains précoces de Strasbourg (Bas-Rhin, France). Étude des structures et du mobilier, Gallia 2013, 70-2, 59-89.

Mayer-Reppert, Petra : Römische Funde aus Konstanz. Vom Siedlungsbeginn bis zur Mitte des 3. Jahrhunderts n. Chr. Fundber. Baden-Württemberg 27, 2003, 441-554. 
Metzler, Jeannot : Das treverische Oppidum auf dem Titelberg. Zur Kontinuität zwischen der spätkeltischen und der frührömischen Zeit in Nord-Gallien, MNHA, Luxemburg, 1995.

Metzler, Jeannot / Gaeng, Catherine : Goeblange-Nospelt. Une nécropole aristocratique trévire, MNHA, Luxemburg, XIII, 2009.

Pauli-Gabi, Thomas : Ausgrabungen im Gebiet der spätlatènezitlichen Befestigung von Vindonissa. Ein Vorbericht zu den Ergebnissen der Grabung Römerblick 2002-2004 (V.002.11), in : Jahresber. Ges. Pro Vindonissa 2004, S. 13-39

Poux, Matthieu (dir.) : Sur les traces de César. Militaria tardo-républicains en contexte gaulois, Bibracte 14, 2008.

Reddé, Michel : Où sont passés les castella Drusiana. Réflexions critiques sur les débuts de l'occupation militaire romaine dans le bassin du Rhin supérieur, in : Revue des études militaires anciennes 2, 2005, S. 69-87.

Reddé, Michel (dir.) : Oedenburg. Fouilles françaises, allemandes et suisses à Biesheim et Kunheim, Haut-Rhin, France. Volume 1. Les camps militaires julio-claudiens. Monographien RGZM, 79, 1, Mainz, 2009 (= Oedenburg I).

Reddé, Michel (dir.) / Schucany Caty / Schwarz, Peter-Andrew : Oedenburg. Fouilles françaises, allemandes et suisses à Biesheim et Kunheim, Haut-Rhin, France. Volume 2. L'agglomération civile et les sanctuaires. Monographien RGZM, 79, 2, 1, Mainz, 2011 (= Oedenburg II).

Schnitzler, Bernadette / Kuhnle, Gertrud (dir.) : Strasbourg-Argentorate. Un camp légionnaire sur le Rhin (ler au IVe siècle après J.-C., Strasbourg, 2010.

Schnitzler, Bernadette (dir.), Brumath-Brocomagus, capitale de la cité des Triboques, Catalogue de l'Exposition, Musée archéologique de Strasbourg (17 avril 2015-31 décembre 2016), 2015.

Schönberger, Hans : Die römischen Truppenlager der frühen und mittleren Kaiserzeit zwischen Nordsee und Inn, in : BerRGK 66, 1985.

Seitz, Gabriele : Villen, Vici, Bäder : die römische Besiedlung des rechten Rheinufers. In : Kelten, Römer und Germanen : frühe Gesellschaft und Herrschaft am Oberrhein bis zum Hochmittelalter, in : Freiburger Universitätsblätter, 159, 2003, S. 47-63.

Spaul, John : Ala ${ }^{2}$, The auxiliary cavalry units of the prediocletianic imperial Roman Army, Andover, 1994.

Speidel, Michael Alexander : Die römischen Schreibtafeln von Vindonissa, Veröffentlichungen der Gesellschaft Pro Vindonissa, XII, 1996.

Waton, Marie-Dominique : Un nouveau système défensif à Strasbourg (Bas-Rhin), in : Rev. Arch. Est et Centre-Est 39, 1988, S. 285-290.

Wendling, Holger : Sasbach am Kaiserstuhl. Siedlungen auf und im Umfeld des Limberges, Kreis Emendingen, in : Kelten an Hoch-und Oberrhein. Führer arch. Denkm. Baden-Württemberg 24, Esslingen, 2005.

Wiegels, Rainer : Ein Reitergrabstein aus dem oberelsässischen Kembs (Haut-Rhin), in : Kleine Schriften zur Epigraphik und Militärgeschichte der germanischen Provinzen, hrsg. von Kresimir Matijevic u. Wolfgang Spickermann, Stuttgart, 2010, S. 566-580.

Wieland, Günther : Die Spätlatènezeit in Württemberg, in : Forsch. u. Ber. Vor-u. Frühgesch. BadenWürttemberg, 63, Stuttgart, 1996.

Zehner, Muriel : La céramique de la Tène finale et du début de l'époque romaine en Alsace, Université de Strasbourg, 2010.

Zehner, Muriel : Habitats de plaine et nécropoles de la Tène moyenne et finale au début de l'époque romaine dans la plaine d'Alsace, in : Bilan scientifique, hors série, 1/2, DRAC Alsace, 2007,

S. 235-242. 
Bereitgestellt von | De Gruyter / TCS Angemeldet

Heruntergeladen am | 22.10.15 09:49 